\title{
EFEITO DE FUNGICIDAS NO CONTROLE IN VITRO DE COLLETOTRICHUM GLOEOSPORIOIDES, AGENTE ETIOLÓGICO DA ANTRACNOSE DO MAMOEIRO (CARICA PAPAYA L.)
}

\author{
Effects of fungicides on the in vitro control of Colletotrichum gloeosporioides, \\ the anthracnosis etiologic agent of the Papaya tree (Carica papaya L.).
}

\author{
Giltembergue Macedo Tavares ${ }^{1}$, Paulo Estevão de Souza ${ }^{2}$
}

\begin{abstract}
RESUMO
Com o objetivo de avaliar a eficiência de alguns fungicidas sobre Colletotrichum gloeosporioides, agente etiológico da antracnose do mamoeiro (Carica papaya L.), testes in vitro foram conduzidos no Laboratório de Epidemiologia/UFLA. Para tanto, utilizou-se o método de incorporação do fungicida ao meio de cultura BDA para avaliação da inibição do crescimento micelial e lâmina escavada contendo água com fungicida para germinação de conídios. Os fungicidas testados foram: azoxystrobin, chlorotalonil, hipoclorito de sódio, imazalil, oxicloreto de cobre, prochloraz, propiconazol, tebuconazol, thiabendazol e tiofanato metílico, nas concentrações de $0,1,10,100,500$ e 1.000 ppm para avaliar a inibição do crescimento do micélio e $0,1,10,50$ e 100 ppm para testar inibição da germinação de conídios. Os fungicidas azoxystrobin, chlorotalonil, imazalil, prochloraz, propiconazol e tebuconazol apresentaram alta eficiência na inibição do crescimento micelial. Os fungicidas dos grupos dos benzimidazóis (thiabendazol e tiofanato metílico) mostraram baixa eficiência e ineficiência, respectivamente, no controle do patógeno, demonstrando uma tolerância do isolado em estudo a estes produtos. O hipoclorito de sódio também apresentou baixa eficiência no controle do crescimento micelial do fungo. Quanto à inibição da germinação dos conídios, os fungicidas que demonstraram maior eficiência em baixas concentrações foram: chlorotalonil e hipoclorito de sódio.
\end{abstract}

Termos para indexação: Colletotrichum gloeosporioides, Carica papaya, controle químico, mamoeiro.

\begin{abstract}
The efficiency of fungicides on Colletotrichum gloeosporioides was evaluated in vitro in the Laboratory of Epidemiology and Diseases Management of the Department of Phytopathology /UFLA. The fungicides were applied either in BDA medium or in glass slide chambers, the latter to assess the effects on conidial germination. The fungicides tested were: azoxystrobin, chlorothalonil, sodium hypochloride, imazalil, copper oxychloride, prochloraz, propiconazol, tebuconazol, thiabendazol and thiophanate-methyl in the concentrations of $0,1,10,100,500$ and $1.000 \mathrm{ppm}$ to evaluate the inhibition of the mycelial growth and in the concentrations of $0,1,10,50$ and $100 \mathrm{ppm}$ to test inhibition of the conidial germination. The fungicides azoxystrobin, chlorotalonil, imazalil, prochloraz, propiconazol and tebuconazol presented high efficiency in the inhibition of the mycelial growth. The fungicides of the benzimidazol group (thiabendazol and thiophanate-methyl) showed low efficiency in the control of C. gloeosporioides. Sodium hypochloride showed low efficiency in the control of the mycelial growth of the fungus. For inhibition of the conidial germination, the fungicides oxychloride, chlorothalonil and sodium hypochloride showed high efficiency, even at low concentrations. C. gloeosporioides showed to be tolerant to the fungicides thiabendazole and thiophanate-methyl, as conidial germination and mycelial growth were not affected in the presence of these products.
\end{abstract}

Index terms: Colletotrichum gloeosporioides, Carica papaya, chemical control, papaya tree.

(Recebido para publicação em 4 de março de 2004 e aprovado em 26 de agosto de 2004)

\section{INTRODUÇÃO}

A antracnose do mamão (Carica papaya L.) é causada pelo fungo Colletotrichum gloeosporioides (Penz), que é o mais importante agente causal de doenças pós-colheita em frutos. O patógeno, além de infectar frutos maduros, pode também infectar frutos imaturos pela penetração direta da cutícula (DICKMAN e ALVAREZ, 1983).
O desenvolvimento do C. gloeosporioides é favorecido por temperatura e umidade relativa elevadas. Seus conídios são liberados e disseminados quando os acérvulos se encontram úmidos. Eles podem ser disseminados por respingo das chuvas, vento, insetos, ferramentas, etc.

Os conídios germinam na presença de água; após a germinação, produzem o apressório e iniciam a penetração no tecido do hospedeiro.

1. Mestre em Fitopatologia pela Universidade Federal de Lavras/ UFLA - Caixa Postal 3037 - 37.200-000 - Lavras, MG.

2. Professor Titular do Departamento de Fitopatologia/UFLA. 
As hifas crescem muito rapidamente, tanto de forma intercelular como intracelular, mas ocorre pouco ou nenhum sintoma nos tecidos. A doença é mais severa quando os frutos começam a amadurecer (AGRIOS, 1998). O fungo sobrevive em pecíolos e folhas velhas, de onde se dissemina pela chuva ou pelo vento, para as flores e frutos novos, e permanece latente até a maturação dos frutos.

Por ser a antracnose uma doença em que os sintomas são percebidos na fase avançada de maturação dos frutos, recomenda-se que o controle seja efetuado antes da fase de maturação.

O principal tratamento indicado para o controle de doenças pós-colheita, no caso de mamão, é o tratamento hidrotérmico $\left(48-49^{\circ} \mathrm{C} / 20\right.$ minutos, em seguida $10^{\circ} \mathrm{C} / 20$ minutos), de acordo com Ventura et al. (2003). Mas, concomitantemente ao uso desse tratamento, recomenda-se a aplicação de ceras e fungicidas para garantir uma maior sobrevida ao fruto.

A diminuição da eficiência dos produtos utilizados comercialmente no controle da antracnose é um fator a ser considerado, uma vez que esse fato é associado ao aparecimento de microorganismos com níveis elevados de tolerância a esses produtos. Diante disso, o objetivo deste trabalho foi avaliar a eficiência dos fungicidas químicos azoxystrobin (Amistar), chlorotalonil (Bravonil utrex), hipoclorito de sódio $(\mathrm{NaClO})$, imazalil (Magnate), oxicloreto de cobre (Recop), prochloraz (Sportak), propiconazol (Tilt), tebuconazol (Folicur), thiabendazol (Tecto) e tiofanato metílico (Cercobin) no controle in vitro do Colletotrichum gloeosporioides, agente etiológico de doenças pós-colheita em frutos de mamão.

\section{MATERIAL E MÉTODOS}

\section{Obtenção do isolado}

$\mathrm{O}$ efeito de diferentes fungicidas no controle do Colletotrichum gloeosporioides foi analisado por meio de testes in vitro, realizados no Laboratório de Epidemiologia e Manejo de Doenças do Departamento de Fitopatologia da Universidade Federal de Lavras, em Lavras, MG.

Os isolados foram obtidos de frutos de mamão da variedade Golden, naturalmente infectados, adquiridos de pomar comercial da propriedade agrícola Algi Frutas, situada no município de Eunápolis, BA. Os frutos foram transportados para o Laboratório de Epidemiologia, onde foram mantidos à temperatura de $25^{\circ} \mathrm{C}$ e diariamente observados para a verificação do desenvolvimento das lesões da antracnose. Após o desenvolvimento das lesões, procedeu-se aos isolamentos retiran- do-se discos de $0,5 \mathrm{~cm}$ de diâmetros nos locais lesionados. Esses discos foram desinfetados em solução de hipoclorito de sódio a $1 \%$ por 2 minutos; depois, colocados em álcool a $40 \%$ por 1 minuto e, em seguida enxaguados com água destilada e esterilizada. Após a desinfestação, procedeu-se a secagem dos discos em papel esterilizado e, logo depois, foram transferidos para as placas de Petri de $9 \mathrm{~cm}$ de diâmetro, contendo como substrato o meio batata dextrose ágar (BDA). Em seguida, procedeu-se a incubação em câmara de crescimento ajustada à temperatura de $22^{0} \mathrm{C}$ e fotoperíodo de 12 horas, até o surgimento da estrutura reprodutiva do fungo, que ocorreu após 4 dias. Depois do aparecimento das estruturas reprodutivas, procedeu-se a repicagem e a transferência para outro meio BDA (GOES 1995).

\section{Bioensaios para avaliar a sensibilidade micelial de C. gloeosporioides aos fungicidas}

O bioensaio foi realizado incorporando-se os fungicidas ao meio de cultura, adotando-se a técnica descrita por Edgington et al. (1971), modificada por Menten et al. (1976). A técnica consiste em dissolver o fungicida em $5 \mathrm{~mL}$ de acetona e completar o volume com água destilada esterilizada até $100 \mathrm{~mL}$, obtendo-se uma solução estoque de 100.000 ppm do ingrediente ativo. A partir da solução estoque, procedeu-se à diluição em série, de tal maneira que cada $\mathrm{mL}$ dessa solução, quando adicionada a $99 \mathrm{~mL}$ de meio BDA fundente $\left(45^{0} \mathrm{C}-50^{0} \mathrm{C}\right)$, produziu a concentração desejada. Após adicionar o fungicida no meio de cultura, realizou-se a agitação para homogeneização dos mesmos. Em seguida, foram vertidos em placas de Petri de $9 \mathrm{~cm}$ de diâmetro. Após solidificação do meio, discos de $7 \mathrm{~mm}$ de diâmetro foram retirados do meio de cultura contendo micélio do fungo, com o auxílio de um vazador e colocados no centro das placas de Petri com fungicidas. As placas foram incubadas em câmara de crescimento, a $22^{0} \mathrm{C} \pm 1{ }^{0} \mathrm{C}$, com fotoperíodo de 12 horas. $\mathrm{O}$ ingrediente ativo dos fungicidas, modo de ação e concentrações, encontram-se na Tabela 1.

$\mathrm{O}$ delineamento experimental utilizado foi o inteiramente casualizado (DIC), com 51 tratamentos e 4 repetições, sendo cada parcela constituída por duas placas de Petri. Os tratamentos foram constituídos pelos seguintes fungicidas: 1.azoxystrobin, 2.chlorotalonil, 3.hipoclorito de sódio, 4.imazalil, 5.oxicloreto de cobre, 6.prochloraz, 7.propiconazol, 8.tebuconazol, 9.thiabendazol e 10.tiofanato metílico em 5 concentrações $(1,10,100,500$ e 1.000 ppm) e a testemunha sem fungicida. 
TABELA 01 - Fungicidas utilizados nos bioensaios do controle in vitro de C. gloeosporioides. UFLA, Lavras-MG, 2003.

\begin{tabular}{lllll}
\hline \multicolumn{1}{c}{ Ingrediente ativo } & Nome comercial & Grupo químico & Tipo & CIA $^{\mathbf{1}}$ \\
\hline Azoxystrobin & Amistar & Estrobirulina & Sistêmico & $500 \mathrm{~g} / \mathrm{kg}$ \\
Chlorotalonil & Bravonil Ultrex & Isoftalonitrila & Protetor & $825 \mathrm{~g} / \mathrm{kg}$ \\
Hipoclorito de sódio & Hipoc. de sódio & Cloro & Contato & $100 \mathrm{~g} / \mathrm{L}$ \\
Imazalil & Magnate & Imidazol & Sistêmico & $500 \mathrm{~g} / \mathrm{L}$ \\
Oxicloreto de cobre & Recop & Inorgânico & Protetor & $840 \mathrm{~g} / \mathrm{kg}$ \\
Prochloraz & Sportak & Imidazol & Contato & $450 \mathrm{~g} / \mathrm{L}$ \\
Propiconazol & Tilt & Triazol & Sistêmico & $250 \mathrm{~g} / \mathrm{L}$ \\
Tebuconazol & Folicur & Triazol & Sistêmico & $200 \mathrm{~g} / \mathrm{L}$ \\
Thiabendazol & Tecto & Benzimidazol & Sistêmico & $485 \mathrm{~g} / \mathrm{L}$ \\
Tiofanato Metílico & Cercobin & Benzimidazol & Sistêmico & $700 \mathrm{~g} / \mathrm{kg}$ \\
\hline
\end{tabular}

${ }^{1}$ Concentração do ingrediente ativo

Avaliaram-se os resultados medindo-se diariamente o crescimento do diâmetro micelial em dois sentidos, perpendicularmente, usando uma régua graduada em milímetros (mm), durante 7 dias após incubação. Os dados foram submetidos à análise de variância $(p<0,05)$ e, quando significativos, à análise de regressão e calculou-se o ED50 (concentração de ingredientes ativo capaz de inibir $50 \%$ do crescimento micelial do isolado). Foi determinada também a concentração mínima inibitória (CMI), ou seja, intervalo entre concentrações dos fungicidas capaz de inibir totalmente o crescimento micelial do fungo.

$\%=\frac{\text { crescimento da testemunha }- \text { crescimento do tratamento }}{\text { crescimento da testemunha }} \times 100$

Após o cálculo do ED50, o isolado de Colletotrichum gloeosporioides foi classificado em 4 categorias de sensibilidade aos fungicidas, segundo a escala de Edgington et al. (1971), em que:

- ED50 < 1 ppm: alta sensibilidade (AS); ED50 1 - 10 ppm moderada sensibilidade (MS); ED50 10 - 50 ppm baixa sensibilidade (BS); ED50 > 50 ppm insensibilidade (I).

Após o cálculo do ED50, os fungicidas foram classificados em 4 categorias de eficiência, de acordo com a escala de Edgington et al. (1971) e Kataria e Grover (1978), citada por Parisi (1997), em que:

- ED50 < 1 ppm: alta eficiência (AE); ED50 1 10 ppm moderada eficiência (ME); ED50 10 - 50 ppm baixa eficiência (BE); ED 50>50 ppm insensibilidade (I).

\section{Sensibilidade de conídios de C. gloeosporioides aos tratamentos}

Para avaliar a inibição da germinação dos esporos, utilizou-se isolado de C. gloeosporioides incubado, por 8 dias, obtendo-se uma densa massa micelial e conidial. Foi obtida previamente uma suspensão de conídios mediante a deposição de $10 \mathrm{~mL}$ de água destilada esterilizada, acrescida de Tween 20 (1 gota $/ 10 \mathrm{~mL}$ de água) sobre a superfície da placa de Petri com micélio fúngico, seguido de fricção das colônias com auxílio de bastão de vidro Drigalski. Após remoção da massa micelial mais conidial, procedeuse a filtragem em camada dupla de gaze esterilizada, obtendo-se uma suspensão de conídios e a sua concentração foi ajustada a $2 \times 106$ esporos $/ \mathrm{mL}$ com auxílio de um hemacitômetro. $\mathrm{O}$ teste adotado foi o da diluição de fungicidas em água destilada e esterilizada, obtendo-se as concentrações de 2, 20, 100 e $200 \mathrm{ppm}$. Amostras de $30 \mu \mathrm{L}$ da suspensão conidial foram depositadas em lâminas escavadas e, em seguida, $30 \mu \mathrm{L}$ da calda fúngica preparada foram depositados sobre a suspensão de conídios e misturados, obtendo-se as diferentes concentrações dos fungicidas com uma suspensão conidial de $1 \times 103$ conídios/mL.

As lâminas escavadas foram colocadas sobre um suporte dentro das placas de Petri de $9 \mathrm{~cm}$ de diâmetro contendo, em seu interior, duas folhas de papel filtro previamente umedecidas com água destilada 
esterilizada e, em seguida, incubadas a $220 \mathrm{C} \pm 10 \mathrm{C}$, durante 12 horas.

Após as 12 horas de incubação, retiraram-se as lâminas escavadas da câmara de crescimento e adicionou-se ácido lático nas lâminas, com o objetivo de inibir a germinação dos esporos após este período. Foram quantificados 100 conídios sob microscópio estereoscópico no aumento de 100x, sendo considerados conídios germinados aqueles que apresentaram tubo germinativo com comprimento de, no mínimo, uma vez o maior tamanho do diâmetro do esporo.

O delineamento experimental foi inteiramente casualizado, com 44 tratamentos e 5 repetições, sendo uma lâmina escavada por parcela. Os tratamentos foram constituídos pelos seguintes fungicidas: azoxystrobin, chlorotalonil, hipoclorito de sódio, imazalil, oxicloreto de cobre, propiconazol, prochloraz, tebuconazol, thiabendazol e tiofanato metílico em 4 concentrações (1, 10, 50 e 100 ppm) e a testemunha em água destilada esterilizada sem fungicida. $\mathrm{Pa}-$ ra análise dos dados, utilizou-se a sobreposição do intervalo de confiança, com $5 \%$ de probabilidade.

\section{RESULTADOS E DISCUSSÃO}

\section{Sensibilidade micelial de $C$. gloeosporioides aos fungicidas}

Os dados referentes à percentagem média de sensibilidade do crescimento micelial dos isolados de $C$. gloeosporioides em diferentes concentrações de azoxystrobin, chlorotalonil, hipoclorito de sódio, imazalil, oxicloreto de cobre, prochloraz, propiconazol, tebuconazol, thiabendazol e tiofanato metílico são apresentados nas Tabelas 2 e 3.

Dentre os fungicidas inibidores da síntese de esteróis, o fungo demonstrou alta sensibilidade ao imazalil, prochloraz, propiconazol e tebuconazol, com ED50 calculado menor que 1 ppm. Resultados semelhantes foram registrados com Colletotrichum gloeosporioides em Citrus (Goes, 1995) e Colletotrichum acutatum em morango (Kososki et al., 2001). Desses quatro fungicidas, apenas o imazalil e o prochloraz são recomendados para o tratamento pós-colheita do mamão. A eficiência comparativa de prochloraz em baixas concentrações sobre o crescimento micelial de C. gloeosporioides foi demonstrada anteriormente por Silva et al. (2001). Os resultados confirmam a eficiência do ingrediente ativo em baixas concentrações, principalmente.

Quando comparados com o fungicida protetor oxicloreto de cobre. Prochloraz mostrou alta eficiência no controle do fungo, apresentando uma concentração mínima de inibição (CMI) entre 1-10 ppm. Os triazóis (propiconazol e tebuconazol) foram também consistentemente eficientes para redução do crescimento micelial do fungo.

TABELA 2 - Equações de regressão, valores médios de ED $_{50}$ (concentração suficiente para inibir 50\% do crescimento micelial), eficiência e sensibilidade de C. gloeosporioides a fungicidas. UFLA, Lavras,MG, 2003.

\begin{tabular}{|c|c|c|c|c|}
\hline Fungicidas & Equações de regressão & $\mathbf{E D}_{50}$ & $S^{1}$ & $\mathbf{E}^{2}$ \\
\hline Azoxystrobin & $\mathrm{Y}=62,941+0,109 \mathrm{X}-0.000078 \mathrm{X}^{2}$ & $<1$ & AS & $\mathrm{AE}$ \\
\hline Chlorotalonil & $\mathrm{Y}=62,834+0,0238 \mathrm{X}$ & $<1$ & $\mathrm{AS}$ & $\mathrm{AE}$ \\
\hline Hipoclorito de sódio & $Y=45,102+0,0319 X-0,000015 X^{2}$ & 381,85 & I & I \\
\hline Imazalil & $Y=69,562-1,107 X^{0,5}+21,277 \log (X)$ & $<1$ & AS & $\mathrm{AE}$ \\
\hline Oxicloreto de cobre & $Y=37,723+0,187 X-0,000131 X^{2}$ & 38,46 & $\mathrm{BS}$ & $\mathrm{BE}$ \\
\hline Prochloraz & $Y=93,189-0,349 X^{0,5}+5,715 \log (X)$ & $<1$ & AS & $\mathrm{AE}$ \\
\hline Propiconazol & $Y=89,773-0,525 X^{0,5}+8,582 \log (X)$ & $<1$ & AS & $\mathrm{AE}$ \\
\hline Tebuconazol & $Y=89,023-0,563 X^{0,5}+9,211 \log (X)$ & $<1$ & AS & $\mathrm{AE}$ \\
\hline Thiabendazol & $Y=38,814-0,923 X^{0,5}+30,583 \log (X)$ & 33,3 & $\mathrm{BS}$ & $\mathrm{BE}$ \\
\hline Tiofanato metílico & $\mathrm{Y}=13,777+0,066 \mathrm{X}-0.000037 \mathrm{X}^{2}$ & $>1000$ & I & I \\
\hline
\end{tabular}

${ }^{1}$ Sensibilidade do C. gloeosporioides ao fungicida

${ }^{2}$ Eficiência do fungicida sobre $C$. gloeosporioides

${ }^{1}$ AS (alta sensibilidade), MS (moderada sensibilidade), BS (baixa sensibilidade), I (insensível)

${ }^{2}$ AE (alta eficiência), ME (moderada eficiência), BE (baixa eficiência), I (ineficiente)

Ciênc. agrotec., Lavras, v. 29, n. 1, p. 52-59, jan./fev. 2005 
TABELA 3 - Valores médios de percentagem de inibição do crescimento micelial de C. gloeosporioides e a concentração mínima inibitória (CMI) ${ }^{1}$.UFLA, Lavras-MG, 2003.

\begin{tabular}{lcccccc}
\hline & \multicolumn{7}{c}{ Concentrações (ppm) } \\
\cline { 2 - 7 } \multicolumn{1}{c}{ Fungicidas } & $\mathbf{1}$ & $\mathbf{1 0}$ & $\mathbf{1 0 0}$ & $\mathbf{5 0 0}$ & $\mathbf{1 0 0 0}$ & CMI \\
\hline Azoxystrobin & 53,7 & 66,1 & 83,2 & 94,8 & 95,9 & $>1000$ \\
Chlorotalonil & 60,4 & 63,2 & 67,8 & 74,7 & 86,4 & $>1000$ \\
Hipoclorito de sódio & 42,9 & 46,2 & 50,1 & 56,6 & 62,4 & $>1000$ \\
Imazalil & 66,9 & 90,5 & 100 & 100 & 100 & $10-100$ \\
Oxicloreto de cobre & 26,2 & 44,6 & 64,7 & 95,1 & 95,1 & $>1000$ \\
Prochloraz & 91,8 & 100 & 100 & 100 & 100 & $1-10$ \\
Propiconazol & 87,7 & 100 & 100 & 100 & 100 & $1-10$ \\
Tebuconazol & 86,8 & 100 & 100 & 100 & 100 & $1-10$ \\
Thiabendazol & 43,3 & 53,9 & 100 & 100 & 100 & $10-100$ \\
Tiofanato metílico & 9,6 & 12,2 & 29,0 & 34,9 & 44,6 & $>1000$ \\
\hline
\end{tabular}

${ }^{1}$ Intervalo entre concentrações, em que podem-se encontrar valores de $\mathbf{1 0 0 \%}$ de inibição do crescimento micelial

A eficiência do propiconazol em reduzir o crescimento micelial in vitro também foi comprovada por Freeman et al. (1997) e Liberato e Tatagiba (2001). A CMI encontrada para o controle do crescimento micelial do $C$. gloeosporioides foi baixa, situando-se entre 1-10 ppm, mostrando assim, a alta eficiência desses fungicidas no controle do patógeno. O fungicida imazalil apresentou alta eficiência no controle do C. gloeosporioides. Resultados semelhantes foram encontrados por Andrivon et al. (1997) com Colletotrichum coccodes em batata. A CMI encontrada para este fungicida situou-se entre 10$100 \mathrm{ppm}$, sendo superior à aquelas apresentadas pelos fungicidas prochloraz, propiconazol e tebuconazol.

Com relação aos fungicidas azoxystrobin e o chlorotalonil, o valor da $\mathrm{ED}_{50}$ para controle do C. gloeosporioides também foi menor que $1 \mathrm{ppm}$, apresentando assim, alta eficiência no controle do patógeno. Os resultados corroboram àqueles encontrados por Picinini e Fernandes (2000), avaliando o efeito desses fungicidas sobre o controle do Colletotrichum lindemuthianum, agente etiológico da antracnose do feijoeiro. Entretanto, Goes (1995) encontrou a $\mathrm{ED}_{50}$ para o controle do $C$. gloeosporioides em citros com chlorotalonil igual a 105 ppm. Quanto à CMI, a concentração para controle do fungo foi entre 1-10 ppm. Para o oxicloreto de cobre, a $\mathrm{ED}_{50}$ foi $38,46 \mathrm{ppm}$, apresentando baixa eficiência no controle do fungo, concordando com Kososki et al. (2001) e Pereira et al. (2003). Quanto ao fungicida thiabendazol, demonstrou baixa eficiência no controle do C. gloeosporioides, sendo a $\mathrm{ED}_{50}$ igual a 33,30 . A tolerância de espécies do fungo aos fungicidas pertencentes ao grupo dos benzimidazóis tem sido observada por Lapeyre de Bellayre e Dubois (1997) e Khan et al. (2001), em isolados de Colletotrichum musae oriundos de fruto de banana. Isso provavelmente deve-se ao uso contínuo deste fungicida pelos produtores para o controle do patógeno. O C. gloeosporioides comportou-se como insensível ao fungicida tiofanato metílico, cuja a $\mathrm{ED}_{50}$ foi superior a $50 \mathrm{ppm}$. A insensibilidade do isolado ao fungicida confirma a presença de resistência cruzada para esse grupo de fungicidas, com grande desenvolvimento micelial na concentração de 1.000 ppm no meio de BDA, confirmando observações de Spalding (1982) com C. gloeosporioides em manga pós-colheita. O hipoclorito de sódio $(\mathrm{NaClO})$ apresentou $\mathrm{ED}_{50}$ de $381,85 \mathrm{ppm}$, isso demonstra insensibilidade do fungo ao fungicida. A CMI foi superior a $1.000 \mathrm{ppm}$, mostrando resistência do patógeno ao $\mathrm{NaClO}$.

\section{Sensibilidade de conídios de C. gloeosporioides aos fungicidas}

Os dados referentes à sensibilidade da germinação de conídios de C. gloeosporioides são apresentados na Tabela 4 . 
TABELA 4 - Percentagem de conídios germinados de Colletotrichum gloeosporioides e intervalo de confiança exato para a variável binomial de conídios.UFLA, Lavras,MG, 2003.

\begin{tabular}{|c|c|c|c|c|c|c|c|c|}
\hline \multirow[b]{2}{*}{ Fungicidas } & \multicolumn{8}{|c|}{ Concentrações (ppm) } \\
\hline & 1 & & 10 & & 50 & & 100 & \\
\hline \multirow[t]{2}{*}{ Testemunha } & $97,60^{1}$ & $\mathrm{f}$ & 97,60 & $\mathrm{~h}$ & 97,60 & $\mathrm{f}$ & 97,60 & $\mathrm{e}$ \\
\hline & $95,82-98,63^{2}$ & & $95,82-98,63$ & & $95,82-98,63$ & & $95,82-98,63$ & \\
\hline \multirow[t]{2}{*}{ Azoxystrobin } & 42,60 & $\mathrm{~b}$ & 31,00 & $\mathrm{c}$ & 23,60 & $\mathrm{c}$ & 17,60 & $\mathrm{~b}$ \\
\hline & $38,33-46,98$ & & $27,10-35,19$ & & $12,71-27,10$ & & $14,51-21,19$ & \\
\hline \multirow[t]{2}{*}{ Chlorotalonil } & 70,40 & $\mathrm{c}$ & 0 & $\mathrm{a}$ & 0 & $\mathrm{a}$ & 0 & $\mathrm{a}$ \\
\hline & $66,25-74,24$ & & $0-0$ & & $0-0$ & & $0-0$ & \\
\hline \multirow[t]{2}{*}{ Hipoc. de Sódio } & 75,80 & $\mathrm{~d}$ & 0 & $\mathrm{a}$ & 0 & $\mathrm{a}$ & 0 & $\mathrm{a}$ \\
\hline & $71,85-79,35$ & & $0-0$ & & $0-0$ & & $0-0$ & \\
\hline \multirow[t]{2}{*}{ Imazalil } & 43,00 & $\mathrm{~b}$ & 23,60 & $\mathrm{c}$ & 0 & $\mathrm{a}$ & 0 & $\mathrm{a}$ \\
\hline & $38,72-47,38$ & & $20,08-27,52$ & & $0-0$ & & $0-0$ & \\
\hline \multirow[t]{2}{*}{ Oxic. de cobre } & 10,00 & $\mathrm{a}$ & 9,60 & $\mathrm{~b}$ & 5,60 & $\mathrm{~b}$ & 0 & $\mathrm{a}$ \\
\hline & $7,66-12,95$ & & $7,31-12,51$ & & $3,89-7,99$ & & $0-0$ & \\
\hline \multirow[t]{2}{*}{ Prochloraz } & 90,20 & e & 88,20 & $\mathrm{~g}$ & 0 & $\mathrm{a}$ & 0 & $\mathrm{a}$ \\
\hline & $87,27-92,51$ & & $85,07-90,75$ & & $0-0$ & & $0-0$ & \\
\hline \multirow[t]{2}{*}{ Propiconazol } & 36,00 & $\mathrm{~b}$ & 23,60 & $\mathrm{c}$ & 0 & $\mathrm{a}$ & 0 & a \\
\hline & $31,91-40,31$ & & $20,08-27,52$ & & $0-0$ & & $0-0$ & \\
\hline \multirow[t]{2}{*}{ Tebuconazol } & 65,60 & $\mathrm{c}$ & 40,60 & $\mathrm{~d}$ & 0 & $\mathrm{a}$ & 0 & $\mathrm{a}$ \\
\hline & $61,33-69,64$ & & $36,38-44,97$ & & $0-0$ & & $0-0$ & \\
\hline \multirow[t]{2}{*}{ Thiabendazol } & 68,60 & $\mathrm{~cd}$ & 57,00 & $\mathrm{e}$ & 46,20 & $d$ & 35,60 & $\mathrm{c}$ \\
\hline & $64,40-72,52$ & & $52,62-61,28$ & & $41,87-50,59$ & & $31,52-39,90$ & \\
\hline \multirow[t]{2}{*}{ Tiof. metílico } & 71,40 & $\mathrm{~cd}$ & 67,00 & $\mathrm{f}$ & 69,20 & $\mathrm{e}$ & 5,64 & d \\
\hline & $67,28-75,19$ & & $62,76-70,98$ & & $65,01-73,09$ & & $52,02-60,69$ & \\
\hline
\end{tabular}

Valores seguidos da mesma letra na vertical não diferem entre si pelo intervalo de confiança $(p \leq 0,05)$

${ }^{1}$ Valores preditos

${ }^{2}$ Intervalo de confiança

Os efeitos dos ingredientes ativos sobre as taxas de germinação de conídios foram diferentes dos observados para o crescimento micelial, refletindo diferentes processos biológicos mensurados a partir destas variáveis. Com relação à inibição da germinação, o fungicida com melhor desempenho na concentração $1 \mathrm{ppm}$ foi oxicloreto de cobre, com 10\% de esporos germinados, seguido pelos fungicidas propiconazol, azoxystrobin e imazalil, com $36 \%, 42,6 \%$ e $43 \%$, respectivamente. Prochloraz, tiofanato metílico e thiabendazole demonstraram maiores percentuais de germinação com 90,2\%, $71,4 \%$ e $68,6 \%$, respectivamente.

Todos os fungicidas, na concentração de 10 ppm, reduziram a germinação de conídios $(\mathrm{p} \leq 0,05)$ em relação à

Ciênc. agrotec., Lavras, v. 29, n. 1, p. 52-59, jan./fev. 2005 
testemunha. Entretanto, os conídios de C. gloeosporioides nesta dosagem apresentaram alto percentual de germinação para os fungicidas prochloraz, tiofanato metílico e thiabendazole. O chlorotalonil e o hipoclorito de sódio apresentaram alto nível de eficiência inibindo 100\% da germinação conidial. Resultado similar demonstrando a eficiência do chlorotalonil sobre o Colletotrichum acutatum, agente etiológico da antracnose em morangueiro, foi encontrado por Kososki et al. (2001). Resultados obtidos por Coutinho et al. (2000), em conídios de Colletotrichum gossypii demonstraram que o hipoclorito de sódio foi eficiente no controle da germinação.

Quando utilizaram $50 \mathrm{ppm}$ dos fungicidas, todos conseguiram reduzir a germinação de conídios comparados ao tratamento testemunha. Dentre estes, os fungicidas tiofanato metílico e thiabendazole mantiveram maiores níveis de germinação, demonstrando tolerância dos conídios do isolado de C. gloeosporioides a esses fungicidas. Os fungicidas imazalil, prochloraz, propiconazol e tebuconazol apresentaram alta eficiência nesta dosagem, inibindo $100 \%$ da germinação dos esporos.

$\mathrm{Na}$ concentração de 100 ppm dos fungicidas, todos foram eficientes em inibir a germinação conidial de C. gloeosporioides, e os tratamentos foram semelhantes significativamente $(\mathrm{p}<0,05)$ aos da concentração de 50 ppm, com exceção dos fungicidas oxicloreto de cobre, thiabendazole e tiofanato metílico. Nesta dosagem, o isolado do fungo manteve-se tolerante aos fungicidas thiabendazole e tiofanato metílico, com alto percentual de germinação dos conídios.

\section{CONCLUSÕES}

O micélio do isolado de C. gloeosporioides em estudo demonstrou maior sensibilidade aos fungicidas azoxystrobin, chlorotalonil, imazalil, prochloraz, propiconazol e tebuconazol.

Os fungicidas do grupo dos benzimidazóis (tiofanato metílico e thiabendazole) mostraram-se ineficientes no controle micelial do C. gloeosporioides e também apresentaram menor inibição da germinação dos esporos na dosagem de 100 ppm.

Os fungicidas chlorotalonil e o hipoclorito de sódio apresentaram inibição total da germinação dos esporos em menor dosagem (10 ppm) em relação aos demais.

\section{REFERÊNCIAS BIBLIOGRÁFICAS}

ANDRIVON, D. et al. Distribution and fungicide sensivity of Colletotrichum cocodes in French potato-producing areas. Plant Pathology, Honolulu, v. 46, p. 722-728, 1997.
AGRIOS, G. N. Plant pathology. Florida: Academic Pressol, 1998. v. 4, p. 817-820.

COUTINHO, M. W. et al. Efeitos de hipoclorito de sódio na germinação de conídios de alguns fungos transmitidos por sementes. Fitopatologia Brasileira, Brasília, v. 25, n. 3, p. 552-555, 2000.

DICKMAN, M. B.; ALVAREZ, A. M. Latent infection caused by Colletotrichum gloeosporioides. Plant Pathology, Honolulu, v. 67, n. 7, p. 748-750, July, 1983.

EDGINGTON, L. V.; KHEW, K. L.; BARRON, G. L. Fungitoxic spectrum of benzimidazoles compounds, Phytopathology, Saint Paul, v. 61, p. 42-44, 1971.

FREEMAN, S. et al. Control of Colletotrichum acutatum in strawberry under laboratory, greenhouse and field conditions. Plant disease, Saint Paul, v. 81, p. 749-751, 1997.

GOES, A. Queda prematura de Citrus: caracterização do agente causal, Colletotrichum gloeosporioides PENZ [Senso ARX, 1957, e controle da doença]. 1995. 16 f. Tese (Doutorado em Fitopatologia) - Escola Superior de Agricultura "Luis de Queiroz", Piracicaba, 1995.

KHAN, S. H.; AKHED, J.; MAGAN, N. Control of the anthracnose pathogen of banana (Colletotrichum musae) using antioxidants alone and in combination with thiabendazole or imazalil. Plant Pathology, Honolulu, v. 50, p. 601-608, 2001.

KOSOSKI, R. M. et al. Efeito de fungicidas em Colletotrichum acutatum em morango e controle da antracnose em morangueiro. Fitopatologia Brasileira, Brasília, v. 26, n. 3, p. 662-666, 2001.

LAPEYRE DE BELAIRE, L. de; DUBOIS, C. Distribution of thiabendazole- resistance Colletotrichum musae isolates from Guadalupe banana plantations. Plant Disease, Saint Paul, v. 81, n. 12, p. 1378-1383, 1997.

LIBERATO, R. J.; TATAGIBA, J. S. Avaliação de fungicidas in vitro e em pós-colheita para controle da antracnose e da podridão peduncular em frutos de mamão. Summa Phytopathologica, Jaguariuna, v. 27, n. 4, p. 409-414, 2001. 
MENTEN, J. O. M. et al. Efeito de alguns fungicidas no crescimento micelial da Macrophomina phaseolina in vitro. Fitopatologia Brasileira, Brasília, v. 1, p. 57-66, 1976.

PARISI, J. J. D. Sensibilidade in vitro de Phomopsis sojae e phomopsis phaseoli f. sp. meridionalis a fungicidas e efeito do tratamento de sementes de soja (Glicine max) inoculados com patógenos. 1997. 61 f. Dissertação (Mestrado em Micologia Agrícola) - Escola Superior de Agronomia “Luis de Queiroz”, Piracicaba, 1997.

PEREIRA, I. S. et al. Avaliação do efeito do sulfato de cobre sobre o crescimento de dois isolados de Colletotrichum spp. em café (Coffea arabica L.). In: CONGRESSO DE FITOPATOLOGIA, 36., 2003, Brasília. Resumos... Brasília: [s.n.], 2003.
PICININI, E. C.; FERNANDES, J. M. Controle químico da mancha angular e da antracnose do feijoeiro. Fitopatologia Brasileira, Brasília, v. 25, p. 92-94, 2000.

SILVA, F. A. N. et al. Controle químico da podridão peduncular de mamão causada por Colletotrichum gloeosporioides. Ciência e Agrotecnologia, Lavras, v. 25, n. 3, p. 519-524, 2001.

SPALDING, D. H. Resistance of mango pathogens to fungicides used to control post-harvest diseases. Plant Disease, Quebec, v. 66, p. 1185-1188, 1982.

VENTURA, J. A.; COSTA, H.; TATAGIBA, J. S. Manejo das doenças do mamoeiro. In: A cultura do mamoeiro: tecnologias de produção. Vitória, ES: Incaper, 2003. p. 231-267. 